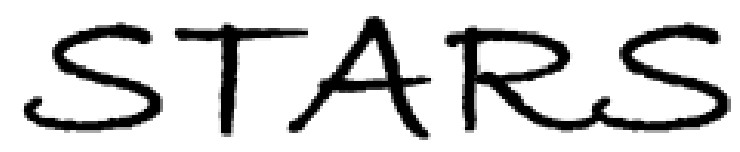

University of Central Florida

STARS

$1-1-2010$

\title{
Scale-dependent anisotropic polarizability in mesoscopic structures
}

\author{
David Haefner \\ University of Central Florida \\ Sergey Sukhov \\ University of Central Florida \\ Aristide Dorgariu \\ University of Central Florida
}

Find similar works at: https://stars.library.ucf.edu/facultybib2010

University of Central Florida Libraries http://library.ucf.edu

This Article is brought to you for free and open access by the Faculty Bibliography at STARS. It has been accepted for inclusion in Faculty Bibliography 2010 s by an authorized administrator of STARS. For more information, please contactSTARS@ucf.edu.

\section{Recommended Citation}

Haefner, David; Sukhov, Sergey; and Dorgariu, Aristide, "Scale-dependent anisotropic polarizability in mesoscopic structures" (2010). Faculty Bibliography 2010s. 213.

https://stars.library.ucf.edu/facultybib2010/213

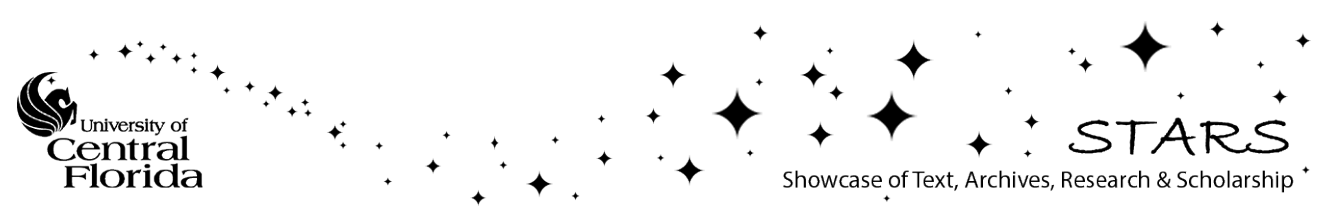




\title{
Scale-dependent anisotropic polarizability in mesoscopic structures
}

\author{
David Haefner, ${ }^{*}$ Sergey Sukhov, ${ }^{\dagger}$ and Aristide Dogariu ${ }^{\star}$ \\ CREOL, The College of Optics and Photonics, University of Central Florida, Orlando, Florida 32816-2700, USA
}

(Received 24 February 2009; revised manuscript received 23 June 2009; published 20 January 2010)

\begin{abstract}
Optical properties of inhomogeneous materials are, in general, scale dependent. We show that, when observed at mesoscopic scales, the local anisotropic polarizabilities depend on the volume of interaction, which may be limited by either the excitation field or material dimensions. We demonstrate the existence of a specific interaction length scale corresponding to the maximum degree of local anisotropy and discuss its relation to the detailed morphology of a disordered medium. Probing these mesoscopic scales provides information about the local structure and allows characterizing material systems that otherwise may appear similar.
\end{abstract}

DOI: 10.1103/PhysRevE.81.016609

PACS number(s): 41.20.-q, 78.20.Bh, 78.67.-n

\section{INTRODUCTION}

In general, material properties are scale dependent, and one can usually define microscopic, mesoscopic, and macroscopic regimes. Their absolute length scales will depend on the specific material characteristics. The microscopic scale refers to the volume over which the material is still homogeneous; probing this scale reveals what is usually referred to as the "intrinsic properties" of the medium. The optical characteristics at the largest, macroscopic scale, involve significant averaging over volumes usually much larger than the characteristic scales of inhomogeneities. All known effective medium theories are based on this averaging principle [1]. Mesoscopic scales, on the other hand, are small enough that fluctuations around the average become important, and may contain additional information about structural morphology.

In the case of inhomogeneous media, a microscopic description provides the intrinsic dielectric properties of the constituents, while a description at macroscopic scales results in an effective averaging of the dielectric properties. For large scale randomly inhomogeneous media, a multiscale description of the light propagation can be envisioned where the Maxwell's equations, the transport equation, and the diffusion equation can be applied to describe the microscopic, mesoscopic, and macroscopic scales, respectively. It should be anticipated that the observed optical response from a light-matter interaction depends on the volume probed experimentally. This concept has been used, for instance, to describe different statistical regimes in near-field scattering from random media $[2,3]$. Note that in the case of near-field microscopy, the volume of effective interaction can be changed by varying the intensity of excitation or by manipulating the tip-sample separation $[2,3]$.

In this paper we will show that, at certain scales, the material response depends strongly on the local structure, and that the individual morphologies of different media result in unique optical signatures. We will exploit the sensitivity of optical interaction to the local material structure, and will identify a specific length scale-maximum anisotropy

\footnotetext{
*dhaefner@creol.ucf.edu

†ssukhov@creol.ucf.edu

†adogariu@creol.ucf.edu
}

length - that depends on the local composition and organization of composite random media. Even though the optical properties in the mesoscopic regime are scale dependant, we will show that this newly introduced length scale represents a unique, intrinsic property characterizing the polarimetric response of optically inhomogeneous media.

\section{LOCAL ANISOTROPIC POLARIZABILITY OF INHOMOGENEOUS MEDIA}

The interaction between constituents of composite materials can generate anisotropic responses, even in situations when both the micro- and macroscopic properties are isotropic. Such structurally induced anisotropy exists, for instance, in aggregates of metal nanoparticle [4] or in small spheres with eccentric inclusions [5-7]. As a result, unique optical signatures develop at these mesoscopic scales.

When the materials' description at mesoscopic scales involves an anisotropic response, one has to go beyond conventional effective medium approaches, which are based on assigning some effective dielectric permittivity to the local properties. In the case of an isotropic distribution of optically isotropic constituents, the effective dielectric permittivity is scalar. However, if the volume of averaging is limited, the local properties can no longer be described with a scalar permittivity. For an averaging volume much smaller than the wavelength, the polarimetric scattering properties can be described as anisotropic Rayleigh scatterers as suggested in Fig. 1. Thus, in this case we can locally assign an effective dielectric polarizability tensor (or, equivalently, permittivity, or refractive index tensors) where the magnitude of the diagonal components, degree of anisotropy, and the orientation of main axes depend on the particular location as suggested in Fig. 1(b) or change from one mesoscopic object to another as illustrated in Fig. 1(d).

In general, the characteristics of these "anisotropic scatterers" will also depend on the mesoscopic volume (level of averaging) considered. Therefore, one can define a local anisotropic polarizability (LAP) that is determined, for example, by the particles dimension if we deal with small inhomogeneous objects Figs. 1(c) and 1(d) or by the excitation volume, as in the case of near-field optical microscopy [2] Figs. 1(a) and 1(b). 
a

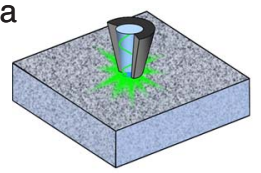

C

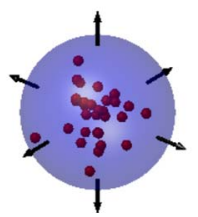

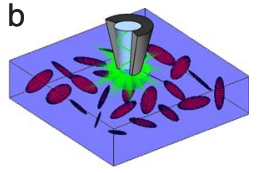

d

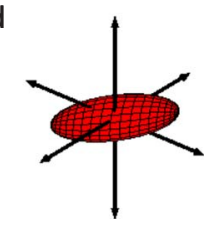

FIG. 1. (Color online) (a) An inhomogeneous material system probed at mesoscopic scales through a local excitation provided by a near-field optical probe. (b) The observed far field response of the material system may be interpreted as being determined by a discrete array of anisotropic Rayleigh scatterers with different local magnitudes and orientations. (c) An inhomogeneous material system where the physical dimensions of the object limit the extents of the field material interaction and the effective anisotropic Rayleigh scatterer (d) producing an equivalent scattered field.

As an example of a mesoscopic optical response, let us consider a spherical interaction volume created either through local excitation or physical extents of the object, with dimensions smaller than the wavelength. Optical inhomogeneities within this volume effectively determine an overall anisotropic polarizability depending on the specific packing structure. In practice, there are different ways to measure this anisotropic polarizability. If the magnitudes of the polarizability elements remain constant during the polarimetric observations, and only the orientation of the effective anisotropic polarizability changes randomly, one can apply the method of stochastic scattering polarimetry (SSP) [8]. Notably, in this case one can recover the anisotropic polarizability irrespective of the incident state of polarization.

There are situations however where the degree of anisotropy may change through the measurement. One can imagine that even for isotropic hard sphere packing, situations may exist where there is some dynamic rearrangement of inclusions resulting in changes of the effective scattering polarizability. In such conditions one can still recover information about the anisotropic polarizability but now it will be in terms of distributions of the tensor elements as we will show in the following.

In a scattering experiment, the relationship between a real polarizability $\alpha$ and the fully polarized incident and scattered fields is commonly described in terms of the corresponding cross-spectral density matrices $W=\left\langle E^{*} E\right\rangle$ as [9]

$$
W_{\text {obs }}=P^{T} \alpha W_{i n c} \alpha P,
$$

where $W_{o b s}, W_{i n c}$ are the cross-spectral density matrices of the scattered and excitation fields, $P$ accounts for the field propagation to the detector, superscript $T$ stands for transpose. To allow for variations in the magnitude of the polarizability not accounted for in the SSP approach, one must measure the entire polarizability tensor and determine both its magnitude and orientation.
To establish simple relationships between the polarizability tensor elements and the measured intensities, a direct procedure can be developed based on sequential excitations. For a fixed orientation of the effective polarizability $\alpha$ there are six unknown elements in the symmetric polarizability tensor. Using Eq. (1), one can find a deterministic relationship for each of the polarizability elements using a scheme based on three independent excitation fields along with a polarimetric detection of scattered intensities. If the three independent excitation fields are chosen to be orthogonal, and if the scattered intensities are measured along the same direction of polarization, one can write a simple expression for the measured intensity $I_{i, j}=\left|\hat{\mu}_{j} \hat{\mu}_{j}^{T} \alpha \mathrm{E}_{i}\right|^{2}$, where $i$ and $j$ correspond to the direction of polarization of excitation field $E$ and the direction of polarization of detection, $\mu_{j}$ is the unit vector along $j$-direction of intensity detection. For example, when the excitation field is polarized along $x$ and the measured intensity is copolarized, the measurement provides directly the $\alpha_{x x}$ component of the polarizability tensor. Following similar steps, a system of six equations can be established and the six independent components of symmetric polarizability tensor can be retrieved. The diagonal form of the polarizability and the corresponding angles of rotation can then be found after performing eigenvalue decomposition,

$$
\alpha=R^{T}\left[\begin{array}{ccc}
\alpha_{a} & 0 & 0 \\
0 & \alpha_{b} & 0 \\
0 & 0 & \alpha_{c}
\end{array}\right] R .
$$

In Eq. (2), the matrix $R$ is composed of the columns of the eigenvectors and accounts for the three-dimensional rotation of the diagonalized polarizability into the detection coordinate frame. The eigenvalues are ordered such that $\alpha_{a} \geq \alpha_{b}$ $\geq \alpha_{c}$.

This procedure of measuring scattered intensities and calculating the diagonal form of the polarizability will now be applied to the case of scattering from inhomogeneous spheres containing inclusions much smaller than the wavelength. We will examine the case of two different structural compositions which, at macroscopic scales, correspond to the same dielectric permittivities according to conventional effective medium theories (for instance, the Bruggeman theory). Inhomogeneous materials were modeled by randomly placing isotropic homogeneous spherical inclusions within the volume of some host characterized by a certain refractive index, and then the optical response was calculated using the coupled dipole approximation (CDA) [10]. The polarizability of these inclusions is related to their assigned refractive indices through Lorentz-Lorenz formula. A Metropolis Monte Carlo (MMC) method of packing inclusions was implemented [11]. To verify the successful packing of hard spheres, the pair correlation function was calculated and compared to the analytical three-dimensional (3D) PercusYevick solution [11]. For each distribution of inclusions, the diagonal elements of the polarizability tensor $\left(\alpha_{a}, \alpha_{b}, \alpha_{c}\right)$ and the angles specifying the orientation of its main axes were determined using the procedure based on Eqs. (1) and (2) which require successive excitation with three fields in different states of polarization and the calculation of corre- 

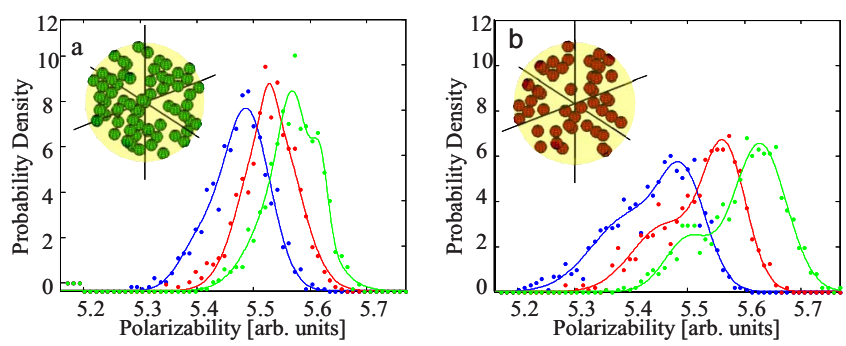

FIG. 2. (Color online) Probability density functions of ordered (see text) diagonal elements of the reconstructed polarizability tensor. The volume of interaction corresponds to a sphere of $50 \mathrm{~nm}$ in diameter having a host refractive index of 1.33 and (a) inclusions with a refractive index of 2 and a volume fraction of 0.27 and (b) inclusions with a refractive index of 2.4 and a volume fraction of 0.18 . The dots are the results of the numerical simulations while the solid lines are guessed "best fit" of numerical data.

sponding scattered intensities in two orthogonal states of polarization. In order to acquire statistically relevant information, a large number of realizations (random packing) were analyzed and the inverse problem of polarizability tensor determination was solved for each realization of the random medium. As a result, we obtain probability density functions (PDF) for the distributions of diagonal elements of the polarizability tensor. The forms of these PDFs reflect the properties of the statistical ensemble of eigenvalues and eigenvectors that characterizes the material properties at this mesoscopic scale.

In Fig. 2 we summarized the results corresponding to the two different structural compositions examined. We note that for the uniformly random material simulated based on a hard sphere model of the inclusion packing, there is no preferential orientation of the effective polarizability. Therefore, our procedure resulted in uniform distributions of the orientations $R$ for both inhomogeneous materials. The values found for the tensor elements on the other hand indicate that, at this mesoscopic scale, the polarizabilities are not only anisotropic, they are also different for the two inhomogeneous materials as can be clearly seen in Fig. 2.

For sample A, the smaller dielectric contrast between the host and the inclusions leads to a narrower distribution of the diagonal elements of the polarizability tensor and, consequently, to smaller fluctuations in the scattered fields. In the case of sample $\mathrm{B}$, however, the larger dielectric contrast results in a larger separation between $\alpha_{a}, \alpha_{b}, \alpha_{c}$ also contributing to larger intensity variations.

To quantitatively characterize the local anisotropic polarizability (LAP), we introduce an anisotropy factor defined as the contrast calculated for diagonal components of polarizability tensor $\alpha$

$$
\begin{aligned}
\Delta & =\frac{\sqrt{3 \operatorname{Tr}\left(\alpha^{2}\right)-\operatorname{Tr}(\alpha)^{2}}}{\operatorname{Tr}(\alpha)} \\
& =\sqrt{2} \frac{\sqrt{\alpha_{a}\left(\alpha_{a}-\alpha_{b}\right)+\alpha_{b}\left(\alpha_{b}-\alpha_{c}\right)+\alpha_{c}\left(\alpha_{c}-\alpha_{a}\right)}}{\alpha_{a}+\alpha_{b}+\alpha_{c}},
\end{aligned}
$$

where $\operatorname{Tr}$ denotes the trace of tensor $\alpha$. Note that, in the past, other definitions have been used for such anisotropy factor. In Ref. [4] for instance, an anisotropy factor $S$ was defined as the variance of depolarization factors $\hat{\nu}(\mathbf{r})=\frac{1}{3} \hat{I}$ $-\int_{V} \hat{G}_{0}\left(\mathbf{r}, \mathbf{r}^{\prime}\right) d^{3} r^{\prime}$. Here $\hat{G}_{0}\left(\mathbf{r}, \mathbf{r}^{\prime}\right)$ is the regular part of the quasistatic free-space dyadic Green's function for electric field, $\hat{I}$ is the unity tensor. In this designation, the local anisotropy factor cannot depend on the excitation volume and, moreover, its locality can be violated in 3D random composites without structural self-similarity, i.e., in nonfractal composites that are of interest here. Another definition of the anisotropy factor was introduced in Ref. [12]; it is similar to Eq. (3) except that it is based on the variance rather than the contrast of the polarizability components. The definition of $\Delta$ in Eq. (3) is most appropriate for our discussion, which focuses on describing the form anisotropy and not necessarily on the absolute magnitude of a specific dipole moment.

Using the definition in Eq. (3), the anisotropy factor $\Delta$ was calculated for every realization of the localized inhomogeneous volume. In this sense, $\Delta$ is a statistical parameter similar, for example, to the contrast measured in near-field microscopy [2]. Of course, an averaged $\Delta$ can then be calculated from the recovered ensemble of values of this parameter. The average anisotropy factors calculated for the two materials illustrated in Fig. 2 are $9.2 \times 10^{-3}$ and $13.2 \times 10^{-3}$, respectively. The $30 \%$ difference clearly demonstrates that LAP is a parameter that can be used to quantify differences between macroscopically similar media.

\section{SCALE-DEPENDENT LOCAL ANISOTROPIC POLARIZABILITY}

In the preceding discussion, LAP was examined over one single length scale. This situation corresponds to fixed volume of light-matter interaction imposed by the measurement procedure. In case of the two different media presented in Fig. 2, the differences will, of course, diminish as this volume of interaction increases; the two different optical responses will converge toward the same macroscopic value corresponding to an isotropic polarizability tensor. The rate of this convergence however may be different depending on the specific structural morphology.

We will turn now our attention to LAP's dependence on the volume of interaction. We have repeated the previous analysis for spheres of different radii $R$ and the results are presented in Fig. 3 where we compare the anisotropy factor $\Delta$ for the case of two different sizes of spherical inclusions randomly distributed within probing volume of different sizes. The main observation is that $\Delta$ always attains a maximum that defines a new length scale characterizing the electromagnetic interaction. This maximum anisotropy length (MAL) represents the length scale over which the response of inhomogeneous medium is most sensitive to the polarization (vector) properties of the excitation field. In other words, it is at this scale that, in average, the depolarization of light during scattering occurs more effectively. Along with scattering mean free path and transport mean-free path that describe the way the energy is transferred, MAL represents another interaction-specific length scale that characterizes the propa- 


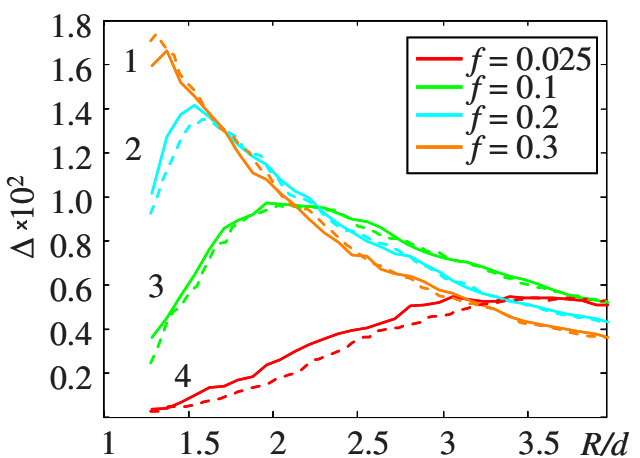

FIG. 3. (Color online) Effective anisotropy factor $\Delta$ as a function of excitation volume $R$ normalized by inclusion diameter $d$ for spherical inclusions with a refractive index of 1.5 randomly distributed in vacuum. The continuous lines correspond to inclusions with diameter $\lambda / 32$ while the dashed lines correspond to inclusions of diameter $\lambda / 64$. Curves 1 to 4 correspond to a volume fractions of inclusions of $0.3,0.2,0.1$, and 0.025 , respectively.

gation of polarized fields through random media.

At length scales smaller than MAL, the local polarizability becomes isotropic with $\Delta$ tending to zero as the probing volume decreases. At larger scales, the macroscopic behavior is gradually approached leading again to an effectively isotropic scattering volume with $\Delta=0$.

As can be seen in Fig. 3, the values of the anisotropy factor appear to be independent of the size of inhomogeneities. This happens, because, in our example, the interaction inside the inhomogeneous volume considered is mostly within the electrostatic regime. Therefore, the behavior of $\Delta$ does not depend on the wavelength and is fully scalable with inclusions' dimensions. Also noticeable in Fig. 3 is the faster decay of $\Delta$ for higher volume fractions of inclusions inside the sphere of interaction. This can also be easily explained by realizing that, for a given excitation volume, the larger number of inclusions corresponding to a higher volume fraction represents in fact a more isotropic medium.

In the particular case when the spherical inclusions can be considered as packed hard spheres, we found that MAL has a simple interpretation. As illustrated in Fig. 4, in this case MAL defines the volume containing, in average, three inclusions. Note that three inclusions represent the minimum number of particles necessary to form a fully anisotropic scatterer. Thus, the statistical averaging for scatterers containing more than three inclusions results in a gradual decrease of the anisotropy factor.

Of course, the other factors determining the optical response of a composite medium are the intrinsic properties of the components. It is expected that, in general, higher anisotropy factors will characterize materials with increasing dielectric contrasts. This is evident in Fig. 5 were we plot the value of the maximum anisotropy $\Delta_{\max }$ as a function of dielectric contrast of inclusions The calculations also demonstrate that the values of $\Delta_{\max }$ simply scale with the magnitudes of dielectric functions of components indicating that the $\Delta_{\max }$ behavior is determined only by the material's structure and not by its composition.

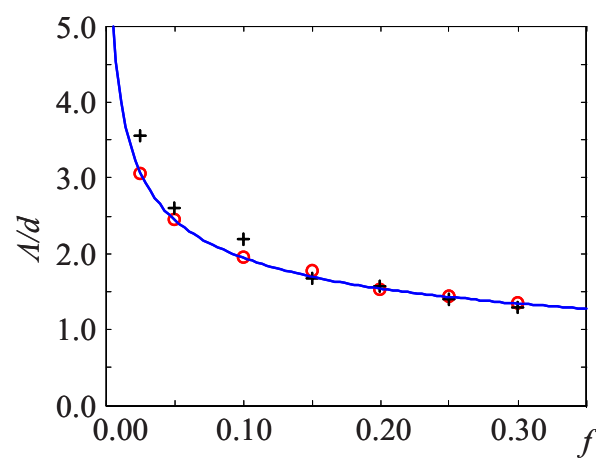

FIG. 4. (Color online) Maximum anisotropy length $(\Lambda)$ normalized by the diameter of inclusions $d$ versus the volume fraction of inclusions $f$. Open circles and crosses represent MAL values corresponding to inhomogeneous media with inclusions of diameters of $\lambda / 64, \lambda / 32$, respectively. The solid line corresponds to the volume containing on average 3 inclusions.

\section{CONCLUSIONS}

We have demonstrated that at mesoscopic scales, the optical response of random media consisting of optically isotropic components may be interpreted in terms of local anisotropic polarizabilities. We illustrated this concept using the example of an inhomogeneous medium containing spherical inclusions, but the model is valid for arbitrary, macroscopically isotropic inhomogeneous media. At mesoscopic scales, different materials can be characterized by their specific anisotropic polarizabilities even though they may have similar effective dielectric permittivities when described in terms of an effective medium approach.

We have also shown that material properties at mesoscopic scales depend on the volume of interaction, in this respect, LAP is an attribute of the electromagnetic fieldmatter interaction. However, a characteristic length scale, maximum anisotropy length (MAL), exists at which the degree of local anisotropy $\Delta$ reaches its maximum. At this scale the inhomogeneous materials are most sensitive to the polarization of incident light. Thus, electromagnetic wave interaction on this scale length results in the maximal depolarization. Along with other characteristics length scales such as the scattering mean-free path, the value of MAL reflects essential intrinsic properties of random media.

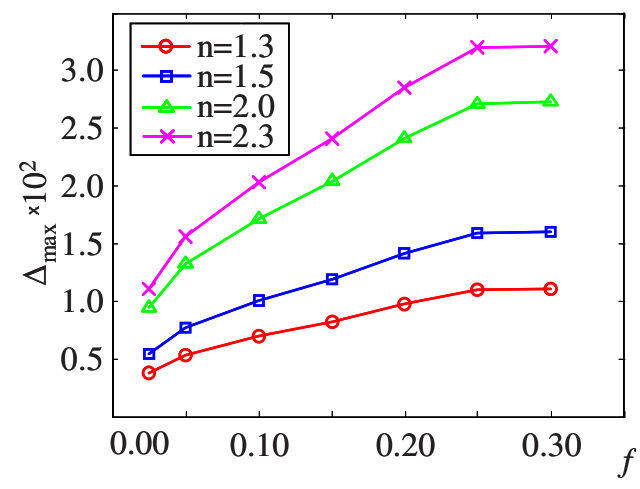

FIG. 5. (Color online) Maximum of anisotropy factor $\Delta_{\max }$ as a function of volume fraction $f$ of inclusions with $50 \mathrm{~nm}$ in diameter and having different refractive index contrasts. 
There are, of course, different means for characterizing the local optical properties of inhomogeneous media. For instance, the scalar contrast of scattered intensities measures the relative variations of the scattering cross-section within the interaction volume $[2,3]$. When this volume increases, the scattered intensity variations decay monotonically to zero with a rate depending on the medium's properties. In this case however, only asymptotic scales can be determined which may affect the specificity. MAL on the other hand is not only derived from a tensorial feature of the material but it is also a local property. Its value is a basic characteristic of material's morphology.

We have also shown that, in the case where the composite material consists of spherical inclusions in a hard sphere packing, MAL may have a purely geometrical representation, not depending on the dielectric properties of the medium's components. In the case of random packing of spheres, we have demonstrated that finding the maximum anisotropy length allows determining the size of the volume containing in average three inclusions. Our findings may also be relevant to the design of novel materials because this new electromagnetic interaction scale represents the material scale at which the polarimetric response of a medium is most sensitive to the excitation field.

Finally, to reconstruct local anisotropic polarizability, we used a method based on three different excitations of investigated mesoscopic volume. However, simpler approaches can be elaborated using the fact that the main axes of anisotropic polarizability may be uniformly oriented in space. Further details will be published elsewhere.

This work was partially supported by the Air Force Office of Scientific Research and by the Army Research Office.
[1] A. Sihvola, Electromagnetic Mixing Formulas and Applications, IEE Electromagnetic Waves Series (The Institute of Electrical Engineers, London, 1999).

[2] A. Apostol, D. Haefner, and A. Dogariu, Phys. Rev. E 74, 066603 (2006).

[3] A. Apostol and A. Dogariu, Phys. Rev. E 72, 025602(R) (2005).

[4] S. V. Karpov, V. S. Gerasimov, I. L. Isaev, and V. A. Markel, Phys. Rev. B 72, 205425 (2005).

[5] G. Videen, P. Pellegrino, D. Ngo, J. S. Videen, and R. G. Pinnick, Appl. Opt. 36, 6115 (1997).

[6] V. Griaznov, I. Veselovskii, P. Di Girolamo, B. Demoz, and D. N. Whiteman, Appl. Opt. 43, 5512 (2004).

[7] F. Borghese, P. Denti, R. Saija, and O. I. Sindoni, J. Opt. Soc.
Am. A 9, 1327 (1992).

[8] D. Haefner, S. Sukhov, and A. Dogariu, Phys. Rev. Lett. 100, 043901 (2008).

[9] L. Mandel and E. Wolf, Optical Coherence and Quantum Optics (Cambridge University Press, New York, 1995), Chap. 6.

[10] B. T. Draine and P. J. Flatau, J. Opt. Soc. Am. A Opt. Image Sci. Vis 11, 1491 (1994).

[11] L. Tsang, J. A. Kong, and K. H. Ding, Scattering of Electromagnetic Waves: Numerical Simulations (Wiley- Interscience, New York, 2001).

[12] K. D. Bonin and V. V. Kresin, Electric-Dipole Polarizabilities of Atoms, Molecules and Clusters (World Scientific, Singapore, 1997). 